\title{
Editorial
}

\section{Evaluating aortic dissection: when is coronary angiography indicated?}

Transoesophageal echocardiography (TOE), rapid sequence spiral computed tomography with intravenous contrast (CT), and magnetic resonance angiography (MRA) have become increasingly useful in the evaluation and diagnosis of acute dissection of the ascending aorta. These diagnostic modalities are fast, safe, non-invasive, and highly accurate, with sensitivity and specificity approaching $100 \%{ }^{1}$ And although most surgeons agree that aortography is no longer necessary before surgical intervention for acute aortic dissection, the role of coronary angiography remains controversial.

\section{Clinical presentation}

Acute dissection of the ascending aorta is a highly lethal event. Without surgical intervention, $50 \%$ of these patients die within the first 24 hours, $75 \%$ die within two weeks, and $90 \%$ die within one month. ${ }^{2}$ Most patients with acute aortic dissection present with sudden onset of severe chest pain that may migrate to the neck or back. Occlusion of aortic branch vessels may cause stroke, abdominal pain, or limb ischaemia. A history of hypertension, known thoracic aortic aneurysm, or characteristics of Marfan's syndrome should alert the physician to the possibility of aortic dissection. On arrival to the emergency room, these patients may appear pale, cool, or clammy, and hypertension is typically present. Hypotension or profound shock usually indicates a catastrophic complication, including pericardial tamponade, aortic rupture, coronary artery obstruction, or severe aortic regurgitation.

\section{Diagnostic modalities}

Echocardiography should be used as the initial diagnostic procedure in patients with suspected aortic dissection. Initially, a transthoracic echocardiogram (TTE) is performed. With this examination, the dissection can be identified and the tear site in the aorta may be located. Left ventricular function, aortic and mitral valve function, regional wall motion, and coronary artery involvement are also assessed. In addition, complications of dissection can be demonstrated, including aortic regurgitation, pericardial effusion, and cardiac tamponade. While TTE provides limited views of the aortic arch and descending aorta, TOE provides excellent images of these structures.

Spiral CT with intravenous contrast allows for identification and evaluation of the aortic dissection and demonstrates any involvement of aortic branch vessels. In addition, a CT scan may identify pericardial fluid, thrombosis of the false lumen, periaortic haematoma or aortic rupture.

Magnetic resonance angiography produces images of the aorta in multiple planes and provides information on flow properties within the aorta. Current MRA technology can distinguish blood from thrombus, differentiate between the true and false lumen, determine location and extent of dissection, and define origins of aortic branch vessels. MRA may also be used to evaluate ventricular function and identify aortic regurgitation.

Although aortography is used less frequently in the evaluation of aortic dissection, it is still useful when vascu- lar occlusion or malperfusion is suspected. The limitations of aortography are well known: it is time consuming and invasive, it requires contrast material, and it may not demonstrate the dissection when the false lumen is thrombosed.

\section{Diagnostic strategy}

The diagnostic strategy for evaluation of acute aortic dissection varies according to location of the dissection, patient symptoms, and patient stability. ${ }^{34}$ In patients suspected of having a dissection involving the ascending aorta, TTE should be performed initially. Stable patients with findings suggestive of ascending dissection are taken to the operating room and examined further with TEE. If TTE findings are equivocal, however, a CT scan is obtained. If a patient presents with symptoms of vascular occlusion or malperfusion, an MRA or aortogram is performed. The haemodynamically unstable patient with acute aortic dissection detected by echocardiography should undergo surgery without further diagnostic delay.

\section{Role of coronary angiography}

Although this strategy for the diagnosis and evaluation of acute ascending aortic dissection has become standard in most institutions, the role of coronary angiography before surgical repair remains controversial. Since coronary artery disease $(\mathrm{CAD})$ remains a major predictive factor for early and late mortality following repair of aortic dissection, ${ }^{5-7}$ it seems logical that coronary angiography and identification of CAD is essential before embarking on major aortic surgery. Ideally, patients with a history of angina or myocardial infarction, a known history of CAD, a recent abnormal thallium image, a history of coronary artery bypass grafting, or acute ECG changes on presentation should undergo cardiac catheterisation before surgical repair of an acute aortic dissection.

Relative indications for coronary angiography

- History of angina

- History of myocardial infarction

- Known coronary artery disease

- Recent abnormal thallium

- Previous coronary artery bypass grafting

- Acute ECG changes on presentation

Cardiac catheterisation in patients with aortic dissection, however, may be technically difficult, time consuming, and potentially risky. Standard femoral or brachial artery cannulation for coronary angiography may not allow access to the true lumen of the aorta. In addition, specific risks of catheterisation include extending the dissection by advancement of the catheter or guidewire, perforation of the aorta by manipulation or injection in the false lumen, or displacement of thrombotic material from a dissected aorta. $^{8}$

Several technical issues should be considered before catheterisation of a dissected aorta. ${ }^{9}$ The arterial access site 
should be carefully chosen after evaluation of the patient's pulses and review of other diagnostic tests. Larger arterial sheaths and guidewires are used to allow better visualisation and torque control. Pigtail catheters should be used initially to enter the left ventricle directly and confirm proper position of the catheter in the true lumen. If successful, pressure measurements are made, the catheter is pulled back to the ascending aorta, and aortography is performed. The aortogram is helpful in defining the shape and size of the aorta and the position and orientation of the coronary ostia. Selective coronary angiography is then performed.

In a report by Griepp and colleagues, 63 patients with either ascending aortic aneurysm (43) or ascending aortic dissection (20) underwent cardiac catheterisation. ${ }^{9}$ Of the 20 patients with aortic dissection, they were unable to engage the coronary ostia in two patients. The only complication in this group of 63 high risk patients was one brachial artery occlusion requiring surgical repair. This report suggests that coronary angiography in patients with aortic dissection is safe, but is it really necessary?

In a recent review by Cohn and associates, 37 consecutive patients with acute ascending dissection were evaluated. ${ }^{10}$ All of the initial 15 patients had coronary angiography and six died $(40 \%)$, three of aortic rupture and none of complications of CAD. Among the next 22 patients, $19(86 \%)$ were operated on without angiography and two died (9\%), neither as a result of rupture or CAD. Furthermore, of the 18 patients that underwent coronary angiography, CAD was found in only two patients (11\%). Based on these data, Cohn and associates suggested that improved survival for patients with acute dissection of the ascending aorta can be achieved by the use of non-invasive diagnostic techniques and that routine angiography may result in delay of surgical intervention and impose greater risk than benefit. Other authors have advocated this approach as well. ${ }^{11}$ Certainly, coronary angiography should not be performed in patients with haemodynamic instability, pericardial effusion, cardiac tamponade, or aortic rupture.

Contraindications to coronary angiography

- Haemodynamic instability

- Pericardial effusion

- Cardiac tamponade

- Aortic rupture

In patients in whom cardiac catheterisation is not performed preoperatively, several intraoperative techniques may be employed to evaluate the coronary arteries.
TOE with colour flow Doppler may be used to evaluate the coronary ostia. Once the ascending aorta is opened, the coronary ostia can be inspected directly, and acute coronary occlusion secondary to the dissection can be corrected. Atherosclerotic occlusive CAD may be suggested preoperatively by ECG findings or wall motion abnormality on TEE, and may be identified intraoperatively by probing, inspecting, or palpating the coronary arteries. If the patient cannot be weaned from cardiopulmonary bypass, then appropriate coronary artery bypass grafting may be attempted.

In summary, patients presenting with suspected acute aortic dissection should be evaluated using non-invasive diagnostic modalities, including echocardiography, CT scanning, and MRA. Aortography is reserved for patients with evidence of vascular occlusion of an aortic branch vessel, and coronary angiography should only be performed in a stable patient with a strong history or clinical suspicion for CAD. This strategy avoids the risks and inherent delays associated with invasive cardiac catheterisation and coronary angiography, expedites the time to surgical intervention, and may decrease the risk of aortic rupture and death.

JEFFREY S MILLER SCOTT A LEMAIRE

Division of Cardiothoracic Surgery JOSEPH S COSELLI

Department of Surgery

Baylor College of Medicine

6560 Fannin, Suite 1100

Houston, Texas 77030, USA

email:jcoselli@bcm.tmc.edu

1 Nienaber CA, von Kodolitsch Y, Nicolas V, et al. The diagnosis of thoracic aortic dissection by noninvasive imaging procedures. $N$ Engl $f$ Med aortic dissection

2 Hirst AE Jr, Johns VJ Jr, Kime SW Jr. Dissecting aneurysms of the aorta: a review of 505 cases. Medicine 1958;37:217-19.

3 Coselli JS, Koksoy C. Aortic dissection. In: Franco KL, Verrier ED, eds. Advanced therapy in cardiac surgery. Hamilton, Ontario: BC Decker, Inc, 1999:296-310.

4 Coselli JS. Surgical treatment of acute and chronic thoracic aortic dissections. In: Ernst CB, Stanley JC, eds. Current therapy in vascular surgery, 3rd ed. Philadelphia: Mosby, 1995;309-14

5 Svensson LG, Crawford ES, Hess KR, et al. Dissection of the aorta and dissecting aortic aneurysms. Circulation 1990;82(suppl IV):IV-24-38.

6 Crawford ES, Kirklin JW, Naftel DC, et al. Surgery of acute dissection of ascending aorta. $\mathcal{F}$ Thorac Cardiovasc Surg 1992;104:46-59.

7 Crawford ES, Svensson LG, Coselli JS, et al. Aortic dissection and dissecting aortic aneurysms. Ann Surg 1988;208:254-73.

8 Hart WL, Berman EJ, LaCom RJ. Hazard of retrograde aortography in disHart WL, Berman EJ, LaCom RJ. Hazard of retrograde
secting aortic aneurysms. Circulation 1963;27:1140-2.

9 Israel DH, Sharma SK, Ambrose JA, et al. Cardiac catheterization and selective coronary angiography in ascending aortic aneurysm or dissection. Cathet Cardiovasc Diagn 1994;32:232-7.

10 Rizzo RJ, Aranki SF, Aklog L, et al. Rapid noninvasive diagnosis and surgi$\mathrm{cal}$ repair of acute ascending aortic dissection. 7 Thorac Cardiovasc Surg 1994;108:567-75.

11 Kern MJ, Serota H, Callicoat P, et al. Use of coronary arteriography in the preoperative management of patients undergoing urgent repair of the thoracic aorta. Am Heart f 1990;119:143-8. 